\section{P07.03 'MYTCELL': A SMARTPHONE APPLICATIONGUIDES LOGISTICSAND MANAGEMENT OF CAR T-CELL \& BITE RELATED TOXICITIES}

\footnotetext{
1,2,3V Blumenberg* ${ }^{*}$, 1,2 L Siegmund, 1,3 $\mathrm{L}$ Fröhlich, 1,3 $\mathrm{M}$ Bergwelt, 1,2 $\mathrm{V}$ Bücklein,

1,3,2 M Subklewe. 'University Hospital, LMU Munich, Munich, Germany; ${ }^{2}$ Gene Center of the LMU Munich, Munich, Germany; ${ }^{3}$ German Cancer Consortium (DKTK) and German Cancer Research Center (DKFZ), Heidelberg, Germany
}

\subsection{6/jitc-2021-ITOC8.44}

Background Bispecific T-cell engagers (BiTE) and CD19-specific Chimeric Antigen Receptor (CAR) T-cell products are approved for relapsed and refractory B-cell neoplasms. However, rapid disease progression and the pre-treatment workflow during manufacturing challenges several specialities of health care professionals and involves a well educated team in the in-patient and out-patient setting. In addition, CARs and BiTEs are accompanied by a new spectrum of immune related toxicities. Currently, clinical trials investigate the safety of outpatient CAR T-cell administration, requiring high-level care during the early post-infusion period. To support the optimal management of these patients, we developed the interactive smartphone application 'myTcell', which guides and educates physicians in the pre-treatment logistics of CARs and BiTEs and management of related toxicities.

Materials and Methods We initiated a multi step content development process with an extensive literature research of toxicity guidelines consented by the ASTCT, SITC, NCCN and EBMT as well as of officially released drug information. Findings were translated into an information platform with diagnostic and therapeutic recommendations as well as algorithms for interactive toxicity grading tools. A prototype has been validated at five Geman treatment centers through a questionnaire, which measures the advantage over common guideline practice. 'myTcell' will become available as medicinal product class I for iOS, Android and desktop in Europe on $15^{\text {th }}$ of July. App development has been funded through educational grants by Celgene, Gilead Sciences, Janssen and Novartis.

Results 'myTcell' guides disease and product specific in a step by step process through the clinical workflow of cell therapy. This includes recommendations for patient screening, safety assessment and stopping rules prior to leukapheresis and CAR T-cell transfusion. Upon entering relevant clinical data for grading of CRS, ICANS and HLH interactive tools display toxicity grade or likelihood of toxicity as well as grade-specific management. Further, 'myTcell' assists with the diagnosis and treatment of pancytopenia and infections. A map visualizes the availability of CAR T-cell therapy in Germany and links inpatient and out-patient care. Besides, 'myTcell' includes an overview of important publications and refers directly to respective PubMed abstracts.

Conclusions 'myTcell' has the potential to become a highly usable smartphone app supporting the application of T-cell recruiting immunotherapies as well as the assessment and treatment of novel immunotoxicities. In addition, it facilitates outreach and connects treatment centers and referrig physicians. Thus, 'myTcell' can translate into increased guideline adherence, accelerated broader and safer application of CARs and BiTEs and improved patient outcomes.

Disclosure Information V. Blumenberg: B. Research Grant (principal investigator, collaborator or consultant and pending grants as well as grants already received); Significant; Novartis,
Gilead Sciences, Janssen, BMS/Celgene. L. Siegmund: None. L. Fröhlich: None. M. Bergwelt: None. V. Bücklein: None. M. Subklewe: None.

\section{P08 Combination Therapy}

\section{P08.01 RATIONALE OF USING THE COMBINATION OF ANTI-PD- 1 ANTIBODY AND ANTI-IL-8 ANTIBODY FOR THE PANCREATIC CANCER TREATMENT}

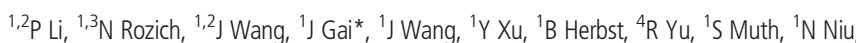
${ }^{1} \mathrm{~K}$ Li, ${ }^{1} \mathrm{~V}$ Fune, ${ }^{1,5} \mathrm{~A}$ Osipov, ${ }^{1,6} \mathrm{C}$ Wolfgang, ${ }^{4} \mathrm{M}$ Lei, ${ }^{7} \mathrm{~T}$ Liang, ${ }^{1} \mathrm{~L}$ Zheng. ${ }^{1}$ Johns Hopkins School of Medicine, Baltimore, MD, USA; ${ }^{2}$ The First Affiliated Hospital, School of Medicine, Zhejiang University, Hangzhou, China; ${ }^{3}$ University of Oklahoma, Oklahoma City, OK, USA; ${ }^{4}$ NovaRock, Biotherapeutics Ltd., Ewing, NJ, USA; ${ }^{5}$ Cedar-Sinai Medical Center, Los Angeles, CA, USA; ${ }^{6}$ New York University, New York, NY, USA; ${ }^{7}$ the First Affiliated Hospital, School of Medicine, Zhejiang University, Hangzhou, China

\subsection{6/jitc-2021-ITOC8.45}

Background Pancreatic ductal adenocarcinoma (PDAC) does not respond to immune checkpoint inhibitors (ICI) therapy as single agent treatments including anti-PD-1 antibody. One of the mechanisms for the resistance of PDAC to ICI is now attributed to the immunosuppressive microenvironment (TME) in PDAC. Myeloid cells are thought to be the predominant immunosuppressive cells in the TME. Human interleukin-8 (IL-8) is a pro-inflammatory chemokine in the CXC family and has the capability of recruiting myeloid cells into the TME to promote tumor progression and immune escape. Therefore, several anti-IL-8 blockade antibodies were developed including HuMax-IL8 and B108-IL8, which both are fully human IgG1 kappa monoclonal antibodies. We therefore tested whether anti-IL-8 antibodies can potentiate anti-tumor activity of anti-PD-1 antibody in a humanized model of PDAC.

Materials and Methods We reconstituted the immune system of the NGS mice with ex vivo activated human T cells and a combination of CD14+ and CD16+ myeloid cells after the mice were orthotopically implanted with human PDAC cells. 10x single nuclei RNA-Seq data processing was further performed to analyze differentially expressed genes among certain cell clusters.

Results Our results showed that anti-PD-1 antibody alone had a minimal anti-tumor activity when mice was reconstituted with ex vivo activated T cells. Interestingly, the infusion of the combination of CD14+ and CD16+ myeloid cells together with anti-PD-1 antibody resulted in a modest anti-tumor activity. Adding either HuMAX-IL8 or B108-IL8 led to a significantly enhanced anti-tumor activity. Both CD14+ and CD16+ myeloid cells appeared to be needed for the full anti-tumor activity of IL-8 blockade because mice infused with only CD14+ myeloid cells did not respond to IL-8 blockade and mice infused with only CD16+ myeloid cells responded partially to IL-8 blockade. This result suggested that the target of IL-8 is mainly present in CD16+ myeloid cells and is likely to be granulocytes. Tumor infiltrating immune cells were isolated and demonstrated that IL-8 blockade increases CD45 +CD11b+CD15+CD14- myeloid cells, which is known to comprise neutrophils and granulocytic myeloid derived suppressive cells (G-MDSC), in the tumors. Reconstitution of the mice with myeloid cells led to a decrease of CD8 $+\mathrm{T}$ cells in the tumors; however, IL- 8 blockade brought the CD8 $+\mathrm{T}$ cell 
number back to the baseline. Consistent with an effect of IL-8 blockade on the increase of CD15+CD14- myeloid cells, single nuclear RNA sequencing analysis of the tumor tissues showed that the innate immune response and cytokine response pathways in the myeloid cell cluster were activated by IL-8 blockade.

Conclusions This result suggested that IL-8 blockade did not simply inhibit myeloid cells as previously anticipated, but potentiated myeloid cells for the innate immune response and concomitant production of type I cytokines. Such immune responses may subsequently activate the effector $\mathrm{T}$ cells as the single nuclear RNA sequencing analysis demonstrated enhanced activation signals in the $\mathrm{T}$ cell cluster from the tumors treated by anti-IL- 8 antibodies. Taken together, this study supports further testing of anti-IL- 8 antibodies including B108-IL8 and HuMax-IL8 in combination with anti-PD-1 antibodies for PDAC treatment.

Disclosure Information P. Li: None. N. Rozich: None. J. Wang: None. J. Gai: None. J. Wang: None. Y. Xu: None. B. Herbst: None. R. Yu: A. Employment (full or part-time); Significant; NovaRock. S. Muth: None. N. Niu: None. K. Li: None. V. Fune: None. A. Osipov: None. C. Wolfgang: None. M. Lei: A. Employment (full or part-time); Significant; NovaRock. T. Liang: None. L. Zheng: B. Research Grant (principal investigator, collaborator or consultant and pending grants as well as grants already received); Significant; BristolMeyer Squibb, Merck, iTeos, Amgen, NovaRock, Inxmed, Halozyme. E. Ownership Interest (stock, stock options, patent or other intellectual property); Significant; Alphamab, Mingruzhiyao. F. Consultant/Advisory Board; Significant; Biosion, Alphamab, NovaRock, Akrevia/Xilio, Ambrx, Novagenesis, Datarevive, Snow Lake Capitals, Mingruzhiyao. Other; Significant; Aduro Biotech.

\section{P08.02 CCR2/CCR5 DUAL-ANTAGONIST ‘LICENSES' THE RADIATION-INDUCED EFFECTOR T-CELL INFILTRATION IN THE ANTI-PD-1 ANTIBODY-TREATED PANCREATIC ADENOCARCINOMA}

${ }^{1,2} \mathrm{~J}$ Wang*, ${ }^{2} \mathrm{M}$ Tun Saung, ${ }^{2} \mathrm{~K}$ Fujiwara, ${ }^{2} \mathrm{~N}$ Niu, ${ }^{2} \mathrm{~A}$ Narang, ${ }^{2} \mathrm{~J} \mathrm{He},{ }^{2} \mathrm{~L}$ Zheng. ${ }^{1}$ The first affiliated hospital of Zhengjiang University, Hangzhou, China; ${ }^{2} J o n h s$ Hopkins university, school of medicine, Baltimore, MD, USA

\subsection{6/jitc-2021-ITOC8.46}

Background The resistance of pancreatic ductal adenocarcinoma(PDAC) to immune checkpoint inhibitors(ICIs) is mainly attributed to the immune-quiescent nature of its tumor microenvironment(TME). Radiotherapy(RT) activates innate responses including the RAGE and TLR2/4 pathways and subsequently modifies the TME by promoting the release of chemokines that recruit inflammatory cells into the TME. In this preclinical study, we examined the PDAC vaccine or RT as a T-cell priming mechanism together with BMS-687681, a small molecule dual-antagonist of CCR2 and CCR5(CCR2/5i) as an immunosuppressive TME-targeting agent, in combination with the anti-PD-1 antibody( $\alpha$ PD1) as a new treatment.

Materials and Methods The hemi-spleen and Orthotopic mice model were used to investigate both GVAX and RT as T-cell priming agents in combination regimens that included $\alpha \mathrm{PD}-1$ and CCR2/5i. Dissected orthotopic pancreatic tumors were collected for analysis of tumor-infiltrating immune cells by flow cytometry. RNA from tumor-infiltrating immune cell pellets and whole-exome RNA sequencing was performed for further mechanism research.

Results CCR2 and CCR5 are associated with the immunosuppressive TME of PDAC patients and their expression were induced after treatment with GVAX+nivolumab. Using a mouse model of PDAC, we demonstrated that the addition of GVAX to CCR $2 / 5 i+\alpha$ PD-1 combination therapy did not significantly improve antitumor activity. However, RT followed by $\alpha \mathrm{PD}-1$ and prolonged treatment with CCR $2 / 5 \mathrm{i}$ conferred significantly better antitumor efficacy compared to the other combination treatments we studied. The combination of RT, $\alpha \mathrm{PD}-1$, and CCR2/5i enhanced intratumoral effector and memory T-cell infiltration. This combination suppressed Treg, M2-like TAM, and M-MDSC infiltration, but not M1-like TAM and PMN-MDSC infiltration. Finally, RNA sequencing showed that CCR2/5i partially inhibited RT-induced TLR2/ 4\&RAGE signaling, which would have otherwise led to the release of immunosuppressive cytokines including CCL2 and CCL5. The inhibition of TLR2/4\&RAGE signaling permitted the expression of effector T-cell chemokines such as CCL17 and CCL22.

Conclusions This study thus supports the clinical development of CCR2/5i in combination with RT and ICIs for PDAC treatment.

Disclosure Information J. Wang: None. M. Tun Saung: None. K. Fujiwara: None. N. Niu: None. A. Narang: None. J. He: None. L. Zheng: None.

\section{P08.03 NEOANTIGEN CANCER VACCINE AUGUMENTS ANTI CTLA-4 EFFICACY}

${ }^{1,2}{ }^{2}$ Palombo*, ${ }^{2} E$ Salvatori, ${ }^{2} \mathrm{~L}$ Lione, ${ }^{1} \mathrm{M}$ Compagnone, ${ }^{3} \mathrm{~A}$ Conforti, ${ }^{1} \mathrm{~L}$ Aurisicchio. ${ }^{1}$ Neomatrix, Rome, Italy; ${ }^{2}$ Takis biotech, Rome, Italy; ${ }^{3}$ Evvivax, Rome, Italy

\subsection{6/jitc-2021-ITOC8.47}

Background Immunotherapy based on anti CTLA-4 ( $\alpha$ CTLA-4) and anti PD1 ( $\alpha$ PD1) is being tested in combination with different therapeutic approaches including other immunotherapy approaches such as neoantigen cancer vaccines (NCV). Here we explored, in two cancer murine models, different therapeutic combinations of $\alpha$ CTLA-4 and/or $\alpha$ PD1 with a plasmid DNA vaccine expressing neoantigens and delivered by electroporation (EP).

Materials and Methods To evaluate the impact of NCV in the MC38 and in the CT26 tumor model three plasmid vaccines were generated with or without CD4 epitopes. Therapeutic DNA vaccines were delivered by EP in different therapeutic protocols including large tumors. Flow cytometry was utilized to measure CD8, CD4, T-reg, and B cells as well as neoantigen-specific immune responses, which were also measured by IFN- $\gamma$ ELIspot.

Results Immune responses were augmented in combination with $\alpha$ CTLA4 but not with $\alpha$ PD1 in the MC38 tumor model with significantly impacting tumor growth. Similarly, neoantigen-specific $\mathrm{T}$ cell immune responses were observed in the CT26 tumor model where large tumors regressed in all mice treated with $\alpha C$ TLA- 4 and NCV. In line with previous evidence, we observed an increased switched memory B cells in the spleen of mice treated with $\alpha$ CTLA- 4 alone or in combination with $\mathrm{NCV}$. 